\title{
Phenomenology of Incompleteness: from Formal Deductions to Mathematics and Physics
}

\author{
Francis Bailly \& Giuseppe Longo \\ Physique, CNRS, Meudon \& LIENS, CNRS-ENS et CREA, Paris \\ http://www.di.ens.fr/users/longo
}

\begin{abstract}
Summary. This paper is divided into two parts. The first proposes a philosophical frame and it "uses" for this a recent book on a phenomenological approach to the foundations of mathematics. Gödel's 1931 theorem and his subsequent philosophical reflections have a major role in discussing this perspective and we will develop our views along the lines of the book (and further on). The first part will also hint to the connections with some results in Mathematical physics, in particular with Poincaré's unpredictability (three-body) theorem, as an opening towards the rest of the paper. As a matter of fact, the second part deals with the "incompleteness" phenomenon in Quantum physics, a wording due to Einstein in a famous joint paper of 1935, still now an issue under discussion for many. Similarities and differences w.r. to the logical notion of incompleteness will be highlighted. A constructivist approach to knowledge, both in mathematics and in physics, underlies our attempted "unified" understanding of these apparently unrelated theoretical issues ${ }^{1}$.
\end{abstract}

\section{Part I. Revisiting "Phenomenology, Logic, and the Philosophy of Mathematics" 2}

Constructivism is the most common philosophical attitude in the mathematics (and practice) of Computing and this in contrast with the prevailing debate in mathematical circles still ranging from Platonism to Formalism. But, what do we mean, today, by "conceptual construction", in the broadest sense? Phenomenology may provide one possible answer to this, by a deeply renewed understanding of Weyl's (and Brower's) ideas, in a perspective close to Husserl's philosophy. Tieszen's book proposes a critical account of modern views in the foundations of mathematics, which is of direct concern for the logician and

\footnotetext{
${ }^{1}$ To appear in Deduction, Computation, Experiment (Lupacchini ed.), Springer, 2008.

2 Part I is a largely expanded version of a review of [Tieszen05], which appeared in Metascience, a review journal in Philosophy of Science, 15.3, 615-619, Springer, 2006.
} 
the theoretician in natural sciences who wants to reflect on the constructive principles of the mathematical intelligibility of the world. We will refer to this book to go further and motivate a broadening of the notion of "construction" as given in formal deductions and arithmetical computations, in either classical or intuitionistic frames. By this broadening, we will understand the incompleteness phenomenon as a "gap" between mathematical construction principles and formal proof principles, following and further devellopping some ideas hinted in [BaillyLongo06].

Tieszen's perspective is original, as the Philosophy of mathematics has been largely dominated by a contraposition between Ontologism and Nominalism, as recalled above. This separated the foundational analysis of mathematics both from our lifeworld and from other scientific domains, including physics where mathematics has a constitutive role. By correlating foundational issues in mathematics and physics, along the lines of [BaillyLongo06], we will try to recompose the foundational break, at least as for the issue of incompleteness.

\section{Part I.I}

The first part of Tieszen'book is dedicated to an introduction to a Husserlian perspective in the foundations of mathematics. It is interesting per se, as a broad survey of Husserl's phenomenology. This is made possible by the relevance that Husserl himself gave to Logic and mathematics in his philosophy of knowledge: writings on Logic and Arithmetic are among the earliest of Husserl's and the related issues accompany his lifelong work.

The constitution of ideal objects, in Husserlian terms, is based on a clear distinction between the transcendental perspective and psychologism. It is the human subject who makes science possible, yet the common endeavour of the historical community should not be confused with the individual analysis: epistemology is a genetic analysis, provided that history is not understood in the usual limited sense, explains Husserl in the "Origin of Geometry" (1933). There are different types and levels of consciousness, which allow the historical dynamics of knowledge: science is built up from the lifeworld experience of human subjects on the basis of active abstraction, idealization, reflection, formalization. The objectivity of knowledge is a constructed one, a result of the interaction by an active subject, beginning with "kinaesthesia", in a living body, in everyday world of life. Meaning is not the passive interpretation of independent signs, but it is constructed in this interaction, it is the result of a "friction" and of structuring of this very world by our attempts to give sense to it; meaning is the result of an action. Of course, we dare to add, this must be understood in a broad sense: Quantum Mechanics for example seems to owe little to kinaesthesia. Yet, it is a paradigmatic case where meaning is the result of active consciousness, beginning with the preparation of the experiment or of the technical context for insight: we are conscious of a quantum object as a constituted phenomenon. Our lived body is just expanded by instruments 
which, in turn, result from a theoretical commitment: this is the richest form of interaction in the sense mentioned above, with no meaningful object without active knowing subject. A (conscious) intentional process is at the origin of this form of knowledge.

As Tieszen explains, consciousness, for Husserl, is consciousness of something. It could be an ideal object of mathematics. The later being the result of a formation of sense founded on underlying acts and contents, which make possible the ideal construction. We would like to exemplify by considering Euclid's action on space by ruler and compass. This action organises figures in space by rotations, translations and reflections, to put it in modern terms. A dialogue with the Gods for sure, but also active measure of ground. But, how to define and measure surfaces, a technique that, in its mathematical generality, is the key Greek invention? In order to conceive exact metric surfaces one has to conceive lines without thickness; there is no way to give a mathematical sound notion of surface, without first proposing, with Euclid's clarity, lines with 0 thickenss. Then, as a consequence of intersecting lines, one obtains dimensionless points, as Euclid defines them (a remark by Wittgenstein). These extraordinarily abstract concepts, point, line etc. are the idealized result of a praxis of measure of surfaces and access to the world by translating and rotating ruler and compass, far away, but grounded on sensory experience. Rotations, translations and symmetries are "principles of (geometric) constructions", a notion to be often used in the sequel. In Euclid's geometry they are used in proofs and they define the geometric objects as given by invariant properties w. r. to these transformations.

Tieszen stresses several times the relevance of invariants in Husserl's foundational approach. "Mathematical objects are invariants that persist across acts" carried out in different contexts. The practical constructions of mathematics, in human space and time, are also stabilized by language and, then, by writing, says Husserl: their constituted ideal nature is primarily the result of their invariance, as conceptual constructions, with respect to suitable transformations of context. And this is extremely modern: invariant structures and transformations were first the foundational core of Riemann's geometry, in Klein's approach, then of Category Theory. Husserl seems to precede the underlying philosophy of Category Theory by his analysis of mathematical knowledge. These invariants are then the essences and, thus, provide the only possible ontology for mathematics; they are the result of different "fulfilled mathematical intentions", as constructions. And these constructions have a horizon, the space of the historical praxis which leaves as trace the most stable invariants of all our mental practices, the structures and objects of mathematics. Then, underlines Tieszen, "truth is within this horizon" as there is no, for Husserl, absolute mathematical truth nor evidence. Yet, mathematical theories are not arbitrary creations (consider the example of Greek geometry above), they are no conventional games of signs: we do not solve open problems by convention, as they are the result of a meaningful and motivated 
construction. The genetic analysis gives evidence for the objectivity of the constitution of mathematics, in the interface between us and the world.

One of the challenges of this approach, that stresses the exactness and stability of mathematical structures, is the correlation with the inexact (i.e. morphological) nature of everyday sensory objects. Even more so, it is the challenge of the relation of mathematics to the sciences of life, where the stability may be global but is not due to exactness. We will go back to this at the end.

Tieszen dedicates a section to the "Origin of Geometry". Even if the significance of that essay is very often present in Tieszens book, we think that it is not sufficiently stressed. This mature text of Husserl's is a splendid progress and a revision for his overall philosophy of mathematics. It radically departs from the partial proximity one can find in Husserl's early books with Frege's logicism and even Hilbert's formalism. However, in this short section, Tieszen presents very clearly Husserl's view on the role of bodily action, by the kinaesthetic and orientation systems, in organising space (the distance of an object is the evaluation of the movement needed to reach it, says Poincaré). Abstraction is then seen as "limitation of attention" and reflection as "adoption of a theoretical attitude" (one can see here the path towards the abstract and reflected nature of our science, which begins with Euclid's Geometry, the original mathematics). Measure, in order to be exact, requires ideal shapes, as given by the notions of point, line etc we mentioned above. These are invariants that found knowledge, from Euclid up to Einstein's Invariantentheorie (the early name of Relativity Theory). Riemann's Geometry, which was born to understand gravitation, underlies this late developments, where, jointly to Category Theory, one sees "invariance and objectivity go hand in hand", since "invariance is a cornerstone of rationality and science". The challenge is to propose, at the same time, the right transformations that preserve the invariants, i. e. to give the right Category (or metric phase-space, to put it in physical terms).

\section{Part I.II}

The second part of Tieszen's book is largely devoted to an effort to find some Husserl in Gödel's philosophy. Gödel, in the last part of his life, became, apparently, a close reader of Husserl. But, Gödel's reading seems more concentrate on some of the writings by Husserl of the beginning of the century and does not seem to span the mature reflection of Husserl, typically to the "Origin of Geometry", in landmark of Husserl's foundational reflection on Mathematics. In particular, Gödel stresses the possible ontological understanding of Husserl's phenomenology far away from the theory of invariance we sketched above. He proposes in particular an identification of physical and mathematical objects, made not in the ground of a similar construction of objectivity, but in reference to a similar objective and autonomous existence. Of course, the foundation of 
physical knowledge is strictly related to the mathematical one, but this common foundation may be understood, in phenomenological terms, by reversing bottom-up Gödel's realism: their simultaneous constitution is the result of a common praxis, not of a similar transcendent reality. Quantum physics is the highest example for this: an electron is a solution of Dirac's equation and nothing else; but Dirac's equation is given on the grounds of robust empirical evidence, prepared by a theoretically oriented acting subject. Here is the virtuous circle of knowledge, which is hypothetical - principles driven, where these principles are grounded on a friction, on a "reality", whatever it may mean, which opposes frictions or resists and canalises our endeavour towards knowledge construction.

So, if one reads Gödel the other way round (both mathematical and physical objectivity - and objects, in a sense - are constituted, including the book and the table mentioned by Gödel), then this is (modern) phenomenology, otherwise one stays with the current interpretation of Gödel as an ordinary Platonist in mathematics. It is not by chance that this interpretation is prevailing, as most published writings of Gödel largely favour this understanding. Of course, the table and the book in Gödel's working place, pre-exist the knowing subject, as they are the result, both the object and the concept (of table, of book), of previous human activities. But also the mountain, which is out there, for sure, is delimited and given a name, isolated from the context, by our historical endeavour towards organising the world (where is it its lower bound, exactly? We draw the mountain's contours). For Gödel, instead, concepts are abstract objects, which exist independently of our perception of them, "perceived" by some physical "ad hoc" organ, which allows intuiting the essence. The lesson instead we learn from (the late) Husserl gives even to intuition the structure of a constituted: intuition in not an absolute, it is the result of our historical praxis, beginning with our phylogenetic history. More closely to us, the mathematician's intuition of the continuum, for example, 150 years after the splendid construction of Cantor's, is deeply indebted to the Cantorian real line: we see the continuum in that way and it is very hard to appreciate different continua (Leibniz's insight, for example.)

In short, Gödel's philosophy of mathematics contains transcendence and very little transcendental constitution, in spite of Tieszen's claim. And Tieszen rightfully recalls Merleau-Ponty's stress on the change towards a theory of existence as preceding essence in the late Husserl, in "Krisis" in particular: existence as invariance and as a result of a constitution. It is a process of free variation that gives the conceptual stability of mathematical (or more generally, conceptual) rules and structures. And this process is similar in mathematics and in physics; while for Gödel it is static perception that is similar in mathematics and physics, as if Physical knowledge (years after Quantum Mechanics) were based on sense perception. Perception, both everyday, common perception, and organised one, is far from static. Even vision requires an activity (saccades at least): perception is the result of an action, which, by its variations, singles out stabilities and invariance. Late unpublished (or 
recently published) reflections by Gödel show an increasing appreciation of the dynamic of thought in Husserl and the role of transcendental constitution in his philosophy. But this unknown writings had little or no relevance in the major influence that Gödel has had in the formation of the platonist approach to foundations. And a philosophy matters also for the effect that it has.

As a matter of fact, the modern perspective in phenomenology even more radically departs from Gödel's blend of realism and idealism: by, perhaps, forcing slightly Husserl (and surely beyond Tieszen's approach), we can even say that any constitution is contingent, as it is the result of a history and a praxis (a praxis in this world, with its frictions to our action).

Whatever is the true teaching by Husserl, our strong stand here is the opposite of the Fregean absolutes that still invade Gödel's views. Of course, though, this constitution has a pre-human history: we share with many animals basic counting praxes, appreciation of borders and trajectories. These are invariants of pre-linguistic activities and, thus, partly precede conscious intentionality, in Husserl's sense.

More generally, mathematics is practiced in "our space of humanity", by our "historical communicating community", as Husserl says, not by an individual subject: this adds to it its further, conscious and intersubjective stability. At once, then, one also understands the effectiveness of mathematics, which suddenly becomes reasonable: it follows from this contingency. There is no real line in the world, nor imaginary number i, but we organise the world by these construed concepts, which are strictly conceived by us, against Gödel's view, and are conceived by acting in this world, this is why they are not arbitrary. Mathematics is the result of this contingent friction between us and the world by a complex praxis of "action - abstraction of the action". The memory of a prey's trajectory, say, is already "abstract": it is the retention of a protension, of the inexistent line, preceding the prey to be caught and that the predator traces in advance, by saccades. And later, the concept of line is the result of our symbolic-linguistic culture, by language, drawing and writing, a further stabilization by intersubjective practices. Here lies its objectivity. Clearly, the resulting invariants, both in mathematics and, perhaps, in general conceptual constructions, may be surely transcendental and, once constituted, non-contingent. By this, we mean that they may be invariant w.r. to transformations of reference system, in physics or mathematics, or of humanly possible forms of life. It is the constitution of these transcendental invariants that is contingent.

Similarly, as humans, we constructed this chair and this table, which were not already there, nor was the concept of chair, of table, but resulted, both the object and the concept, from our constructive action and linguistic exchanges. Once constructed, the table, the chair and their concepts stay there, go cross generations and history, through changes, and transcend each individual life. And we also invented language and the alphabet, which are not in the world; yet, by their constitutive history, they are extremely (yet, reasonably) effective in understanding each other and the world, no less than mathematics, in their 
domains of application. They are at least as effective as this chair, which seats us so well, it fits us and the physical world! And we understand each other by talking and by (re-)producing language in writing: where is the miracle? It is no more than in the panglossian nose and spectacles, which surprisingly fit each other so well. And we can even use words with certain prevailing meanings to express very different situations, by metaphors say. That is, we may transfer linguistic expressions and conceptual structures, by formal or semantic drifts, similarly as complex numbers can be used to express conjugate values in Quantum Physics, three centuries after their invention.

In conclusion and against Logicism and Platonism metaphysical necessities, which need miracles and/or inspire an unreasonable effectiveness when transferred to the world, we claim that mathematics is objective and effective, exactly because it is the constructed contingency of maximally stable invariants of our action in space-time and of reasoning, by gestures and language, along our phylogenetic and human history. Or, also, we define mathematics as the set of conceptual practices that is maximally stable and independent from the contingency of their constitutive path (they are invariant under transformations of humanly possible frames, as we said). And this, since Euclid's triangles, which, by definition, do not depend on the thickness of the traits, or the colour of the ink. This maximal stability and independence, which is part of their construction, makes the mathematical concepts more stable than a chair and a table or their concepts; they better go through history.

Finally, let's observe that mathematical objects are limit constructions, obtained by a conceptual "critical" transition (see part 2), where the constitutive contingency is lost at the limit. Euclid's line with no thickness or the "transcendental" number $\pi$ is the result of a geometric construction, pushed to the limit. But, in the end, their objectivity does not depend on the specificcontingent and more or less abstract reference to actual traits or sequence of rational numbers, needed to conceive or present them: at the limit, the transition to infinity provides us with a perfectly stable conceptual object (a mathematical ideality, some like to say). Their value and their sense do not depend on the specific construction or contingent converging sequence of rationals, yet needed to conceive or define them. And the conceptual transition is irreversible: no way to go from $\pi$ to a given specific converging sequence; there are infinitely many of them and $\pi$ is the invariant under transformation (change) of equiconvergent sequence. In the second part we will return at length on this understanding of the constitution of mathematical idealities as a conceptual (we will say "critical") transition, in analogy to the physics of critical phase change.

Let's go back to Tieszen's book. In a very scholarly fashion, Tieszen's chapters on Gödel's allow to grasp the differences between the mature Husserl and Gödel's philosophy, as well as the ontological shift of Gödel's which may at most be referred to Husserl's work at the beginning of the century. Yet, we would continue further to dig into the non-phenomenological reference to absolutes in Gödel's thinking. Tieszen explains how Gödel believed Hilbert to 
be correct in supposing the decidability of all number-theoretic questions, in spite of his own undecidability (incompleteness) theorem: rationality needs just to be extended by new laws and procedures. This rationalistic optimism, a belief in absolute-ideal essences and their possible perfect knowledge, is paradigmatic of how both Formalism and Platonism detached the foundation of mathematics from physics and allows us to understand the opposite views of mathematicians more deeply concerned with the latter (philosophically at least, as Hilbert greatly contributed to Mathematical physics): Poincaré, Weyl, Enriques. How could a leading Nominalist and a leading Ontologist (in an broad semi-Husserlian sense), Hilbert and Gödel respectively, both possibly say that there could be no undecidable assertion? And this when Poincaré had shown that, given a formal system of a few equations (the Newton-Laplace system for three bodies in their gravitational fields, 1890), one could state a (formalizable) proposition, parametrized on a sufficiently distant time, that could be provably shown to be undecidable, under the intended (and best) physical approximation of initial data. This was the first great result on the mathematical unpredictability of non-linear deterministic systems. No added (physically possible) mathematical knowledge can solve this. This is why Poincaré cries out against Hilbert's "sausage machine's view of mathematics" (an aggressive stand recalled also by Tieszen): he has an entirely different Philosophy of Knowledge. Let's analyse this point more closely.

\section{Preliminary reflections on Incompleteness, in mathematics and in physics}

In [Longo01], it was suggested that Poincaré's three-body theorem (see [Barrow97] for an introduction and an historical account) is an epistemological predecessor of Gödel's undecidability result. Of course, Hilbert and Gödel were speaking about purely mathematical 'yes or no' questions, while unpredictability shows up, at finite time, in the relation between a physical system and a mathematical set of equations (or evolution functions). That is, in order to give unpredictability, Poincaré's Negative Result, as he called his proof of the non-analyticity of the equations for three-body system, needs a reference to physical measure. Measure is always, in classical (and relativistic) physics, an interval, that is an approximation, by which non-observable initial fluctuations may give observable thus unpredictable evolutions, in presence of non-linearity of the mathematical determination - a set of equations or an evolution function (main reasons: the initial interval expands exponentially, by the so-called Lyapounov exponents, and it is "mixed", [Devaney89], [Diacu92]). Yet, one can reformulate the problem in terms of a formal trajectory "reaching or keeping away" from a given target neighbourhood: if the deterministic system is chaotic, the mathematical question cannot be decided (see [Hoyrup07], [Hoyrup08] for surveys). However, this is slightly unsatisfactory as it only shows undecidability properties of chaotic dynamics (and not the converse) and it partly relies on abstract properties of real computable 
numbers, instead of intervals, that do not need to fully express the mathematics of physical systems. In particular, an effective measure theory (along the lines of Lebesgues's measure, the locus for dynamic randomness) needs to be introduced. The problem then is to have a sound and purely mathematical treatment of the epistemological issue (and obtain a convincing mathematical correspondence between unpredictability and undecidability).

Now, unpredictability of deterministic systems is randomness in classical physics (see [BaillyLongo07]) and it may also be expressed as a limit or asymptotic notion. Under this form, it may be soundly turned into a purely mathematical issue. That is, randomness, as a mathematical limit property, lives in formal systems of equations or evolution functions, with no need to refer to physical processes and their approximated measure.

On these purely mathematical grounds, the second author conjectured that a rigorous formal link could be shown, between Poincaré's unpredictability and Gödel's undecidability, by passing through Martin-Löf number-theoretic randomness. This is a "gödelian" notion of randomness, as it is based on Recursion Theory and yields a strong form of (strong) undecidability for infinite $0-1$ sequences (an infinite sequence is random if it passes all effective statistical tests, see $\left.[\mathrm{ML} 66]^{3}\right)$. On the side of physical dynamics, its mathematical counterpart can be found in reference to Birkhoff's notion of ergodicity, which refers to infinite trajectories, a purely mathematical approach at the infinite limit, with no need to refer to the interval of physical measure to engeder randomness. That is, mathematical dynamical systems, in their (Lesbesgue) measurable spaces, allow to define generic points and infinite random trajectories, in the ergodic sense, [Petersen90].

Recently, M. Hoyrup and C. Rojas, under Galatolo's and the second author's supervision, proved that dynamic randomness (à la Poincaré, thus, but asymptotically, following Birkhoff), in suitable effectively given measurable dynamical systems, is equivalent to (a generalization of) Martin-Löf randomness. This is a non-obvious result, spreading across two doctoral dissertations (available by summer 2008, see aknowledgements) and gives an indirect, but relevant, we believe, technical link between Gödel's incompleteness as undecidability and Poincaré's unpredictability ${ }^{4}$.

On more philosophical grounds, Poincaré, in several writings, also tried to relate the foundations of mathematics to that of physics, passing by cognition and action in space. Similarly for Weyl and Enriques, who insisted, during their entire life, on a parallel foundation of scientific knowledge in these exact Sciences, see [BaillyLongo06]. We insisted here on the connections between

\footnotetext{
${ }^{3}$ Martin-Löf's randomness, inspired by Kolmogorof's ideas, has been developped by many, Chaitin most remarkably, see [Calude02] for a classic.

${ }^{4}$ Other links betwen physical and algorithmic randomness may be found in the litterature, yet the connection via ergodic theory seems new. In particular, [CaludeStay05] relates algorithmic randomness to quantum indetermination, a different issue; in Part 2 we will discuss the difference between incompleteness and indetermination in Quantum Mechanics.
} 
the issues of decidability and predictability, at the core of mathematical and physical theoretizing. Of course, unpredictability in not in the world, it is not an ontological matter, it concerns the relation of our forms of (mathematical) knowledge to the world and this by the role of physical measure (our form of access to the world). However, as we hinted, mathematics brings it within a purely theoretical realm, by pushing to the asymptotic limit the mathematical treatment of the intended physical processes in space-time continua, following Birkhoff ergodicity. And this relates, as we said, to algorithmic randomness.

Observe now that, since the beginning of the $\mathrm{XX}^{\text {th }}$ century, physicists have been discussing the immense philosophical challenge of the intrinsic indetermination of Quantum Physics (again, an issue related to measure) and that, in 1935, the possible "incompleteness" of Quantum Mechanics was proposed as a key theoretical issue, as we shall see in Part II. These internal limitations to knowledge (physico-mathematical unpredictability, intrinsic indetermination vs. quantum incompleteness), whose understanding opened the way to two major scientific domains, in classical and Quantum physics, were simply out of the scope of the discussion of the two metaphysical rationalisms of Hilbert and Gödel, in foundations.

\section{Back to Gödel's philosophy}

Gödel's philosophy, in our opinion, seems far away from any internal debate in physics such as the one we will discuss in Part II (an example is given by the mathematically remarkable, but physically unsound paper on the circularity of relativistic time, see [Goedel49]). It escapes though trivial physicalism, as for Theory of Mind. As Tieszen explains, Gödel argues against Turing's claim that mental procedures cannot carry any further than mechanical procedure, as both mind (in the brain) and machines are both "finite state devices". To this, Gödel observes that mind is not static, but constantly developing. A very modern view, as it is now clear that there are no "states" in brain/mind, but only processes: Turing's Machines "instantaneous descriptions" simply do not make sense in cognition. In particular, Gödel remarks that abstract meanings or concepts may be the result of limit procedures (to put it short: after a few iterations, we "look at the horizon" and we construct and understand, say, irrational numbers, projective limits, transfinite ordinals.)

In chapters 6 and 7, Tieszen further develops his close analysis of Gödel's path towards phenomenology. This is a rather unaccomplished path, as we explained above and as one may deduce also from Tieszen's many references and comparisons. Yet, this path is very interesting, as it brings Gödel to a clarification of the role of meaning in Arithmetic. With the help of notions from phenomenology, Gödel derives from his incompleteness results the need for a reference to meaning as "categorial intuition" in (arithmetic) proofs. Thought structures or thought contents, in Gödel's words, are the result of insights which go beyond the combinatorial properties of symbols and require a reflection upon the meanings involved. This is what we call the meaningful 
construction principles, as rooted in our cognitive history (from evolution to human history); the well-ordering of integer numbers is an example (see [Longo02]). It is in this sense that formal proof principles are incomplete w. r. to construction principles, see also below. As we recalled, for the late Husserl as well, these meanings are the result of a "formation of sense" which is grounded in our "historical spaces of humanity".

While missing this point about the constitution of meaning and principles, Gödel takes up another fundamental theme of phenomenology: intentionality. In Tieszen's interpretation, Gödel uses and understands it as directedness towards invariants. This fundamental structure of thought allows us to understand even sense perception as a non passive but active and constitutive first step of knowledge building: the early, pre-conceptual, singling out of invariants. Of course, from this perspective, Benacerraf's dilemma or alike, to which Tieszen refers in a highly critical way, are just part of the new scholastic which F. Enriques had forecasted long before ("the Philosophy of Mathematics is heading towards a new scholastic: the Scylla of Ontologism and the Charybdis of Nominalism", he observed in 1937). As a matter of fact and well beyond Quine's Platonism, in particular in Set Theory (meaning as truth is "out there"), Gödel proposes an understanding of meaning as content, that is as a result of intentional processes. Husserl's intentionality again allows understanding categorization, from perception to thought, and thus content as part of meaning formation.

As we are talking of more or less naive forms of Platonism, which regained relevance by, helas, too common interpretations of incompleteness, a reference should be made here to Penelope Maddy's book. Tiestzen begins by some high praises of it and continues with ... an extremely severe critique of any relevant idea in that book. Tieszen explains the misunderstanding of Gödel's refined Platonism which is brought back to the usual flat ontology, in contrast to the phenomenological components that Tieszen showed to contain. He insists on the wrong "bon sens" (our words) attitude of considering that "(some) sets are part of the physical world" and perceived by us as such, without any of the constructed conceptual stability and invariance that is proper to mathematics. This brings Maddy to an unavoidable relativism, which is rather alien, says Tieszen, to that science. There are three eggs, three atoms and so forth and, ho miracle, we have the mathematical "set of three" and the concept of "three"! But mathematics is a science of structures: there is no mathematics without structures. Set Theory originated in the logical foundations of mathematics and passed aside the foundation of modern physics exactly because this refers to geometric invariants or geodetic principles (which are symmetry invariants: they reflect a structure). Mathematics actively organises the world and our forms of scientific knowledge, it shapes them simultaneously, since it is grounded on invariant structures, beginning with perception: the line is not a set of points (let alone a set of eggs), but a gestalt. It may be logically found or reconstructed, a posteriori, as a set of points (Cantor), or even without points, in some toposes (Lawvere, Bell, see [Bell98]). 
Tieszen analysis continues by scholarly and stepwise demolishing Maddy's book (in a very gentle and motivated way, of course, though some polemic tension may be appreciated in between the lines). One further issue should be mentioned here; it concerns the naive attitude of confusing "bon sens" (which is different from "common sense") with "formation of sense": there is a huge gap between the two and this is called the constructed objectivity of science. Science always goes against "bon sens". Greek mathematics did not begin by looking at sets of eggs, but by daring to propose non-existing lines with no thickness and nondimensional points. Modern science began against the "bon sens" evidence of the Sun turning around the Hearth. But even the concept of number is a constructed and complex invariant w.r. to ordering, organizing and "small" counting, as ancient (pre-human?) practices ([Dehaene97] is a classic about this). It is complex, as it is the result of many active experiences of transformations and their invariants.

But then, why not to propose that the unity of consciousness (and mathematical meaning) is due to Quantum entanglement as global effects in the brain? This is the question discussed in chapter 10. Personally, we are not against occasional audacious speculations as the one proposed by Penrose in several bestsellers and analyzed by Tieszen. However, as it is a matter of finding physical phenomena in a material structure (the brain) it would be better, at least in principle, to start from some empirical evidence. Of course, there is none of this and Penrose's starting point is Gödel's incompleteness theorem. And a few assumptions.

First, awareness is a physical action and any (classical) physical action can be simulated computationally (in Turing's sense); then, truth is an absolute matter, to which we have access by awareness. So, again, in order to escape the Scylla and Charybdis of Nominalism and Ontologism, Penrose suggests a shortcut from microphysics to consciousness.

As for the role of Gödel's theorem, unfortunately this is based on a misunderstanding of the proof of it: Tieszen quotes a fine analysis of Penrose's mistakes made by Feferman. The result of this misunderstanding is the belief that one can deduce from it the absolute and transcendent nature of truth. Tieszen criticizes this point not only on the technical grounds of Feferman's remarks but also along the lines of his phenomenological analysis of meanings as intentions. In short, infinity, as meaningful thought structure, constituted along history, steps in the proof of consistency of Arithmetic. This may be more closely understood by an analysis of recent "concrete" incompleteness results, carried on in [Longo02] and, more informally, in [Longo05].

There is no place here to criticize further Penrose's physicalism. It takes explicitly for granted that current physical theories are complete w.r. to the world (this was also Aristotle's opinion): so, if a phenomenon is not classical (relativistic) - thus computable (really? see [LongoPaul08]), it must be describable by some Quantum Theory. If the founding fathers of Quantum physics had had the same attitude, they would have searched in existing theories, Relativity Theory, say, or in variants of (thermo-) Dynamics the solution to 
strange phenomena such as the energy spectrum of the hydrogen or the three bodies' problem of the helium. They proposed instead a radically new theory.

It is interesting to see how often physicists, who in general defend non trivial philosophies of knowledge within their discipline, when looking at life or cognitive phenomena, just claim: this must be understood in terms of (reduced to) one of our theories (usually: the one I know best). Typically, when discussing of General Relativity and Quantum Mechanics they talk of "unification" not of reduction, as many do when referring to biology. Unification, in contrast to reduction, means that one must be ready to invent a new theory that changes radically both pertinent notions of field or even the intended objects (String Theory) or the structure of space-time (Non Commutative Geometry). The point is that in order to "unify" one must have two robust field theories and, in biology, we miss exactly an autonomous and proper notion of "biological (causal) field" (this is discussed at length in [BaillyLongo06]). There is no use to analyse any further Penrose's claim that all classical physical processes are computational, since we know, for example, that even the evolution of our planetary system is provably non computable. As a matter of fact, recent results of unpredictability in deterministic systems, [Laskar94] prove that there is no way to compute the relative positions of all planets and the Sun in more than ten million years. That is, the system will have a position in the continuum of space that no digital machine can compute; this a "concrete" and difficult version of Poincaré's theorem on non-linear Dynamical Systems and their unpredictability, as uncomputability. It is a "concrete" result, similarly as the famous combinatorial results by Paris and Harrington, or Freidman version of Kruskal's theorem, which provide concrete combinatorial example, that is (interesting) propositions about interger numbers, that are provably unprovable in Formal Arithmetic, see [ParisHarr78], [Harrington85] (and [Longo02] for a discussion).

Of course, by this we do not pretend to exhaust the discussion on mind, as developed also in Tieszen's chapter on Penrose: even a sound biological theory of brain would still be far from our symbolic culture and the phylogenetic and historical formation of sense in our "communicating community", which is the place where meaning, consciousness and intentionality are formed. That is, a purely biological theory of brain would be incomplete w.r. to cognition, as this should be embedded in our social and historical "forms of life": brain signs have meaning only within a context. A contingent distinction of theories does not imply dualism, as much as the distinction of quantum field from relativistic one is not dualism, but a distinction of phenomenal levels. And unification is a difficult matter. Yet, Gödel's attitude on the matter, as explained by Tieszen, is a traditional ontological dualism Mind vs. Brain: the first has access to (infinitary) truth and meaning, by a metaphysical ahistorical path, the second should be less complex than our planetary system as, according to Gödel, it is fully computational. Unfortunately, Penrose's answer to this ordinary dualism is highly insufficient. 


\section{Part I.III}

This part of Tieszen's book is largely dedicated to Weyl and Poincaré's philosophies, with a final reflection returning to Frege and Husserl. First, though, a very interesting understanding of Intuitionism is presented. Dummett's approach, in particular, is surveyed, with a clear presentation of his Wittgensteinian constructivism: "meaning is use". Unfortunately, Dummett seems to focus only on linguistic sense of use, in a clear contraposition with the Brouwerian orthodoxy (for Brouwer, mathematics is languageless!). In addition to language, use as action, as gestures, as (imagined) figures and drawings as forms of presence in space and time or of human interaction contribute to the transcendental constitution and to single out invariants by our intentional attitude. In our phenomenogical perspective, meaning goes well beyond the linguistic truth-conditions and brings us back again to intentionality, broadly construed (here we would like to pause and insist, with Merleau-Ponty, that, well before consciousness, "le mouvement et l'action sont l'intentionalite' originaire"). Tieszen stresses another crucial point which is not captured by the linguistic turn: the intentional grasp of meaning doesn't need to be fully determinate nor clear and exact. This allows inserting the phenomenological theory of meaning in human contexts where communication is enriched (and made possible) by polysemy, ambiguity, cross references .... Mathematics, in our approach, singles out and is determined as the locus of maximal stability and invariance among our conceptual constructions: no polysemy is allowed, in principle, no ambiguity: we force a - relative - stability of meaning; this is mathematics.

Tieszen's analysis of Weyl's constructivism is unfortunately limited to his views on the foundations of mathematics. The point though is that both Weyl's and Poincaré's views should not be detached from their Philosophies of Nature, though difficult it may be to spell them out, in particular from the overloaded and unorganised writings by Poincaré. Thus, while it is true that in "Das Kontinuum" Weyl spends several pages in sketching a (mathematically remarkable) predicative theory of reals, however, his short "flirt" with brouwerian intuitionism is motivated by his broader constructivist perspective that always tries to relate the foundations of mathematics to our general "human endeavour towards knowledge". Thus, like Brouwer, but well beyond Brouwer's psychological time, Weyl reflects to the continuum as space-time structure. He distinguishes between space continua and the phenomenal time continuum, the time we experience in consciousness. This cannot be reduced to analytic representations by points, in his view. Tieszen gives a very clear account of Weyl's understanding of time (based on a "specious or extended present") as well as of his short lasting predicativism; yet, the presentation makes an insufficient effort in connecting "Das Kontinuum" and the contemporary work in "Raum-Zeit-Materie". Moreover, later on, Weyl invented gauge theory, in physics, as an analysis of invariance, with a peculiar role of symmetries. Considering the constitutive role of both these notions for the 
foundations of mathematics and physics, one should find also there the rich perspective of Weyl's.

An informal survey of this physico-mathematical unity may be found in "Symmetry", Weyl's last book. Observe also that, while stressing the interest of Brouwer's free choice sequence as an approach to the continuum, Weyl, in several places, radically disagrees with Hilbert's formalist project as "the idea of a potential mechanization trivializes mathematics" (in "Das Kontinumm", where in an early section Weyl hesitantly conjectures the incompleteness of Arithmetic, in 1918! This should be noted more often).

Weyl's reflections are of a rare depth in the $\mathrm{XX}^{\text {th }}$ century Philosophy of mathematics, scattered in several writings, from "Das Kontinuum" (1918) to Weyl's simple, but deep masterpiece that we mentioned, "Symmetry" (1954). One can find there the key role of symmetries in the constructed objectivity of our physico-mathematical knowledge. There is no ontological miracle nor miracle whatsoever, but the role of mathematics as a science structuring the world, by its very definition. One sees, in that book, the classification of planar symmetries (by rotation, translation, reflection: the Greek insight) as the prerequisite for understanding that of finite groups. Other classifications, from Platonic solids to crystals' symmetries are then understood as conceptual, if not technical, consequences of these regularities, which make space-time intelligible and objective. Gauge invariance, a result of rotation/translation symmetries, is also seen as a foundation of Relativity and Quantum Theories (more on symmetries in the foundations of physics and mathematics may be found in [vanFrassen], [BaillyLongo06]). In short, Weyl shows that we singled out from and imposed on the world, also by our own bodily symmetries, a few regularities as tools for knowledge (for understanding, organizing), of which symmetries are the core part, and we called it "mathematics". In some cases, this is exhaustive of our spatio-temporal and linguistic representations: it covers them completely (by classifications). In no way does it follow from this, however, that mathematics can be detached from our own existence in this world and its concrete, active representations; on the contrary, it roots mathematics in them, starting with these resonance of symmetries, between us and the world 5 .

The passage to Poincaré's philosophy is motivated by Tieszen in the best possible way, in our opinion, that is by the call for an epistemological or cognitive dimension of proof. Poincaré refers to proofs by their meaning and geometric organization (against the flat arithmetic coding by Hilbert), and stresses the cognitive grounding of mathematical structures. Proof is the realization of a mathematical expectation, it is grounded on possibly new con-

\footnotetext{
5 "But perhaps this question can be answered by pointing toward the essentially historical nature of that life of the mind of which my own existence is an integral but not autonomous part. It is ... contingency and necessity, bondage and freedom, and it cannot be expected that a symbolic construction of the world in some final form can ever be detached from it." [Weyl49], p.62.
} 
cepts, it requires a conceptual investigation, as in the contentual approach by Martin-Löf quoted by Tieszen. The geometric intuition of the real line precedes, in Poincaré's view, its logical foundation. In general, the analysis of the construction of mathematical concepts and structures (the epistemology of mathematics), which is extraneous to the formalist and logicist (Platonist) approaches, is a matter of a "living process in which the mind remains active": an analysis of human cognition, thus, in our sense. And here Tieszen moves to Husserl's "Krisis", a lesson for today even more than for his time: by "the construction and mastery of formal systems, Science becomes nothing but technique". It loses meaning and "forgets its historical origin". It loses also students, a dramatic process in Western world, as, once that making physical experiments, for example, is transformed into implementing computer simulations (isn't any classical-relativistic physical process computational?), why should one study physics? And many claim that also understanding biology or Cognition is only a matter of good computer models and compilers. Thus, financing increasingly goes only to short term technical and "competitive" industrially oriented projects, possibly producing quick computational models of whatever natural process. This is gradually killing theoretical long-term collaborative construction of knowledge, often grounded on "negative results" such as Goedel's or Poincaré's. In their time, these results were the opposite of positive modelization. It happened though that they opened the way to major scientific areas, the geometry of dynamical systems and computability, whose practical fall-out are under everybody's eyes.

The epistemological relevance of the major negative results we have been talking about goes toghether with their technical interest and actually motivated them. Tieszen's final chapter goes back to Husserl's philosophy in order to single out more closely, from his early writings, the role of meaning and intentionality as constitutive of the epistemological analysis and, in our views, of the analysis of proofs as well (in view of the incompleteness of formalisms). Husserl's reflection may thus be mentioned both for the need to preserve "meaning and sense" to the general scientific enterprise, beyond its technical developements, and for opposing Frege's project of eliminating intuition from proofs (meaning would be "obtruding", according to Frege!).

\section{Conclusion on mathematical incompleteness}

We tried to better understand incompleteness, which is usually seen just as an incomplete covering of semantics by syntax, as the gap between proof principles (on which formal deductions are based) and construction principles (the locus of meaning, in the constitutive relation between mathematics and our life world). Both principles are the result of the contingent constitution we mentioned above, but the former are the late commers of this process and, by principle, they forget the constitutive path. The latter instead may allow a 
reconstruction of the cognitive and historical gestures that lead to them and yield meaning ${ }^{6}$.

This view of principles and incompleteness further specifies Husserl's approach in mathematics and it is even more remote from Frege's view, as he actually believed in Peano Arithmetic's "categoricity" (to put it in modern terms: there is only one model, or syntax coincide wit semantics), a much stronger property than Hilbert's completeness. As we shall see next, the discussion on "meaning" and "interpretation", also in relation to "completeness", is at the core of theoretical and foundational analysis also in physics.

\section{Part 2. Incompleteness and Uncertainty: differences and similarities between Physics and Mathematics.}

In the view of discussing some themes around the notion of incompleteness, such as it appeared with force during the 30 s, a period which saw the flourishing of Gödel's great logical theorems, and such as it started to foster debates and arouse new perplexities in physics during the same era, we will delve here into some concepts of quantum physics.

Firstly, and somewhat trivially, we know that in physics the accumulation of empirical proof does not suffice to account for the totality of the theoretical construction which represents phenomena. This is how, in physics and at a very first level, is manifested the incompleteness of proof principles, as grounded on empirical evidence, relative to construction principles. The latter are principles of conceptual construction and are, often, limit principles. No empirical evidence showed to Galileo that bodies never stop. Yet he dared to propose the principle of inertia at the non-existing limit of absence of friction, which is the only general and pertinent one (cf. [BaillyLongo06] for more on this; chapter 4 for example, shows the constructive role of the geodesic principle).

However, this incompleteness has been thought way beyond this first and simple level, both in classical and in quantum frames.

\section{Completeness/incompleteness in classical theories}

We first return to a conceptual comparison between the positions of Laplace and Hilbert on the one hand and of Poincaré and Gödel on the other. The first two refer to a sort of strict completeness of theories that are physical in one case, and mathematical in the other: they would be complete in the sense that any statement concerning the future would be decidable (this is the Laplacian predictability of systems determined by a finite set of equations) or, regarding logico-formal derivations, the completeness or decidability of arithmetics (or

\footnotetext{
${ }^{6}$ More on this may be found in In [Longo02], [BaillyLongo06] and [Longo05].
} 
of any sufficiently expressive axiomatic theory: this is Hilberts completeness and decidability conjecture).

The other two demonstrate incompleteness: Poincaré, with his theorem on the three-body problem, showed unpredictability of interesting non-linear systems, which we may understand as undecidability of future states. Their dynamics will be said to be sensitive to the initial conditions, and in that, deterministic, yet unpredictable: minor variations, possibly below the level of observability, could cause major changes in the evolution of the system. Gödel proved at once the unprovability of coherence and the intrinsic undecidability and incompleteness of arithmetics (and of all its formal extensions), by constructing an undecidable statement, equivalent to the formal assertion of consistency (which is thus also unprovable within the system). We hinted to the epistemological and the recent technical link between the physico-mathematical problem and the purely mathematical one. It can then be interesting to try to characterize the main types of physical theories in terms of this relationship to completeness.

If relativistic theories may indeed proclaim theoretical completeness in the sense defined above, it very well appears that theories of classical dynamics on the one hand and quantum theories on the other hand, may present two distinct manners by which they manifest incompleteness.

In the case of chaotic dynamic systems, as we observed, unpredictability is associated to the sensitivity to the initial conditions joint to the non-linearity, typically, of the (formal) determination. It may however be observed that a (theoretically) infinite precision regarding the initial data is meant to generate a perfectly defined evolution (deterministic aspect of the system), or that a reinitialization of the dynamic system with rigorously identical values leads to reproducible results. Of course, this in principle and from the mathematical viewpoint, because physically speaking, we are still within the context of an approximation and the result of a measurement in classical and relativistic is always, in fact, an interval and not a unique point, in spite of the supposed mathematically continuous (space-time)background. Hence, we may notice that an essential conceptual transition appears between that which pertains to a finite level of approximation and that which constitutes a singularity, at the "actual" infinite limit of precision (or, one could say, between the unpredictable and the theoretically reproducible).

In quantum theories, contrastingly, as we shall see, be it an issue of relationships of indetermination or of non-separability, unpredictability is intrinsic to the system, it is inherent to it. In this case, the degree of approximation matters little: there is no conceptual break between finite and infinite, since, as for measurement, there is no supposed continuous space-time background (conitnuity may be found in Hilbert spaces, before measurement, but this is a different issue). Another way to observe this consists in noticing that this time, regardless of the rigor of the reinitialization of the system, the results of the measurement will not necessarily be individually reproducible (proba- 
bilistic character of quantum measurement), even if the law of probability of these results can be perfectly well known.

It is randomness then which is at the center of these theories, and this by subtle differences from classical frameworks. This point is delicate and we refer the reader to [BaillyLongo07].

\section{Incompleteness in quantum physics}

In the debate on the completeness of quantum physics, Einstein, Podolski and Rosen (EPR) highlighted three characteristics of the physical object which they believed to be fundamental in order to be able to speak of a complete theory (see [Einstein35], [Bohm51]):

1. the reference to that which they called elements of reality (as existing objects "beyond ourselves", independently of measurement or access, say);

2. the capacity to identify a principle of causality (including in the relativist sense);

3. the property of locality (or of separability) of physical objects.

Bell inequalities, [Bell64], and their experimental verifications, notably by Aspect and his team, [Aspect82], have shown that the third EPR postulate was not corroborated: experience shows that two quantum objects having interacted remain for certain measurements a single object, consequently non separable, regardless of their distance in space. In other words, for two quantons having interacted, even if they are afterwards causally separated in space, any measurement of a value on the one would instantaneously determine the value of the other, against fundamental principles of Relativity Theory.

Presented this way, the eventual incompleteness (or, conversely, completeness) of quantum physics seems to have nothing to do with what is meant by completeness or incompleteness in logic, which have been addressed above ${ }^{7}$. However, a deeper examination reveals that what appears at first glance as a lexical telescoping may not be completely fortuitous.

To each of these characteristics "required" by EPR in physics, one may, indeed, without distorting the significations too much, associate characteristics "required" by mathematics (recall that principles of proof correspond to formal deductions in mathematics and to empirical "evidence" in physics):

1. elements of reality would be put into correspondence with proofs that construct existence, that is, the effectiveness of mathematical constructions (which, axiomatically or not, we have seen the difference, cause mathematical structures to exist - similarly as we construct, isolate or "point-out" objects in physics, see 1 above);

\footnotetext{
${ }^{7}$ By accepting, for this discussion, to use the framework of arithmetics or of set theory (ZFC type) and its models, a framework within which the questions relative to logical completeness were first raised.
} 
2. the principle of causality would be put into correspondence with the effectiveness of the administration of proof (which presents and works upon the rational sequences of demonstrations, be they stemming from formalism as such or not - the deductive chain is here placed in correspondence with the causal one in physics, see 2 above);

3 . the property of locality (or of separability) would be put into correspondence with the autonomy of mathematical theories and structures inasmuch as they would be "locally" decidable (or that within a formal theory, any statement or its negation would be demonstrable, see next for a relation to 3 above).

Now it is precisely this local autonomy of theories, this "locality" in terms of decidability, which seems to be contradicted by the theorems of incompleteness in mathematics. The latter indeed refer to a sort of globality of mathematical theories in that one may need to use stronger principles to prove a statment in a given theory. Techinically, recent results by Friedman show that, relatively simple combinatorial statements of Peano Arithmetic may require increasingly large ordinals or cardinals to be proved ${ }^{8}$. In a sense, the "global" structure of orders, even of the entire mathematics (if one believes that ordinals and cardinals express the proof theoretic power in mathematics) seems to step in the proof of local properties of the first order arithmetic. So, the adjunction of (finetely or recursively many ) axioms to a theory doesn't render "decidable", at most "more expressive" (or capable of deciding previously undecidable statements), but at the cost of generating a new theory which requires the same treatment itself, because, remaining formal, it would still be incomplete. In other words, there is no way to isolate arithmetic nor any other sufficiently expressive mathematics and deduce within it "completely": tools from any other branch of mathematics may be need in a proof of a statement of the given (apparently simple) theory.

But we can probably push the analysis further than suggested by these conceptual analogies.

In mathematics, if we refer to the interpretation of Gödelian incompleteness in terms of discrepancy between construction principles (structural and significant) and proof principles (formal) that is, in terms of incomplete covering, between semantics and syntax (all achievable propositions are not formally derivable, or, more traditionally: semantics exceeds syntax), then a closer relationship may be established. This relationship concerns, among other things, the introduction and the plurivocity of the term interpretation, according to whether it is used in a context of model theory or if it is taken in its common physical sense. In model theory, the excess of semantics (construction principles) with regard to syntax (proof principles) is first manifested in distinct interpretations (existence of non-isomorphic models, that is, non-categoricity) of a same syntax (for example, the non-standard model of Peano's arithmetic, cf. above). Gödelian incompleteness furthermore demon-

\footnotetext{
${ }^{8}$ see http://www.math.ohio-state.edu/users/friedman/ .
} 
strates that some of these models realize different properties (technically, they are elementarely non-equivalent).

In physics, if we accept to see the equivalent of syntax in the mathematical structure of quantum mechanics and the equivalent of semantics in their proposed conceptual-theoretical interpretations (hidden-variable theories, for example, see below), then the "semantics" would also exceed "syntax" and, consequently, a certain form of incompleteness (in this sense) would be manifest. ${ }^{9}$ But more profoundly, in the case of quantum physics, it is the excess of the "possible", the quantum states in a Hilbert space, over the "actual", once states are measured, which best illustrates this type of parallelism and of comparison. In other words, the result of a measurement corresponds to a plurality of potential states leading to it, each with its well defined probability. A sort of non-categoricity of the states (non-isomorphic, even if they belong to the same system) relatively to the well defined result of the measurement. The latter operates here like a sort of "axiomatic" constraint in that it stems, as we have seen, from the physical principles of proof, which are empirical (empirical proofs are "constraint" by physical measure).

The concept of incompleteness was then understood by EPR in the sense that quantum physics should be deterministic in its core and that its probabilistic manifestations would only be due to the lack of knowledge of "hidden variables" and of their behaviors. This actually amounts to saying "there are hidden causal relationships between particles that are not described by the theory". Now, the EPR argument is experimentally contradicted by the violation of the Bell inequalities and the fact that the property of non-separability is indeed inherent to quantum physics. In this sense quantum theory has been shown to be complete (there are no hidden variables).

Can we nevertheless speak of incompleteness in a sense different than that of EPR without however it being totally extraneous? In other words, would it be possible to formulate a proposition that is undecidable in the sense that it would be true according to one model and false according to another? Let's consider the crucial statement which can be attributed to EPR: "there are hidden variables". As we have just seen, this statement is false according to the usual model (standard interpretation) of quantum mechanics. Yet a theory presenting the same properties as quantum mechanics can be constructed, in

\footnotetext{
${ }^{9}$ If we want to continue with the analogy and in parallel with Logic, we will also notice that this search for hidden variables, which prove to be non-local, evokes in a way the method of forcing, which enabled Cohen to demonstrate the independence in ZFC of the Continuum Hypothesis and of the Axiom of Choice (by constructing a model not realizing them, whereas Gödel had constructed a model that does, see [Jech97]). The hidden variables in question are indeed "forcing" for the physical model they nevertheless continue to respect. This is somewhat analogous, conceptually speaking, to the logical situation where forcing propositions are compatibly integrated with the original axiomatic construction: one adds or forces extending variables or properties, previously "hidden" or not assumed.
} 
which this statement is true on condition that an adjective is added: "there are non-local hidden variables". ${ }^{10}$

\section{Incompleteness vs. indetermination.}

Sometimes, confusion is set in between the concept of incompleteness (as presented by EPR) and that of indetermination (as highlighted by Heisenberg). So let's try to further explain how are the relationships between incompleteness and indetermination, which do not cover the same conceptual constructions.

The issue of incompleteness such as raised by EPR leads, we have seen, to the search for "hidden variables" which would "explain" the counter-intuitive behavior of quantum phenomena. As we observed, it is possible to elaborate hidden-variable theories, but these variables are themselves non-local, therefore simply postponing the intuitive difficulties. In this regard, it is better to preserve the canonical version of quantum physics, the Bell inequalities and the experiments by Aspect which highlight the property of quantum non-separability (that for two separated quantons, which have previously interacted, measurement on one would determine the value of the other). By highlighting the fact that one of the origins of this situation stems from the use of complex numbers (in state vectors or wave function) as additive quantities (principle of superposition), whereas that which is measured is a real number and refers to the squares of the modules of these quantities. We will return to this in a moment.

Quantum indetermination ("the uncertainty principle"), for its part, mobilizes somewhat different concepts: it consists in the treatment of explicit variables (non-hidden), such as positions and momentums, of which the associated operators will present a character of noncommutativity (measuring first one observable, then the other does not commute: a fundamental property

10 "Local variables" is an expression which is also equivalent to "variables attached to particles" (they depend only on properties specific to a given particle each: this is locality). To speak then of non-local variables is to express the fact that the value of a variable which governs the behavior of a particle may not only depend on this particle, but may also depend on (remote) other particles. This is also another way to consider the non-separability we have just mentioned. As a matter of fact, the distinction (separability) between two particles having interacted is a representation that stems directly from classical physics, be it relativistic. For its part, quantum physics proves to be fundamentally non-local, that is to contain entangled (non-separable) quanta. Thus, a type of valid proposition in an interpretation (no hidden variables and non-separability) can be false in another (existence of hidden variables, but by specifying non-local variables). In fact, more broadly, the controversy first initiated by L. de Broglie continues among some physicists, currently a small minority, with regards to the character of causal determination, which would be classical but hidden, undescribed by the theory: an incompleteness of quantum physics. 
which will lead moreover to the development of non-commutative geometry, the current insight into the space of microphysics). It is in fact an issue of the constraints which weigh the Plank constant (small but non-null) upon physical measurement, precluding simultaneous measurement with an "infinite" precision of two conjugated magnitudes such as positions and the corresponding momentums, which we have just mentioned.

If we wanted to roughly distinguish the two types of conceptual ambiguity introduced by these quantum properties with regard to habitual intuition, we would say that incompleteness refers rather to an ambiguity of object (is the object local or global? How is it that according to the nature of the experiment it appears to manifest either as a particle or a wave? $\left.{ }^{11}\right)$. Indetermination instead leads to an ambiguity of the "state" of the system: a quantum object of which we know the precise position would be affected by an imprecise velocity; conversely, the precise knowledge of a velocity would entail an "indeterminacy" regarding the position occupied. In a purely mathematical way, a quantum object which we would manage to "stop" would occupy all space.

In fact, both versions of these quantum specificities refer to a difficulty of describing quantum phenomena "classically" within time and space of classical or relativistic theories, while their description within their own "abstract" spaces (a Hilbert space, for instance) is perfectly clear ${ }^{12}$.

Regarding space, we will note several traits which make of our usual intuition of space (and even of the Riemannian manifold of general relativity) an instrument which is unadapted to properly represent quantum phenomenality $^{13}$ :

i) Firstly, as we have indicated above, quantum quantities are defined at the onset on the field of complex numbers $\mathrm{C}$, as opposed to classical and relativistic quantities which are defined at the onset of the field of real numbers R. It stems from this that in quantum physics, what is added (principle of superposition) is not what is measured (complex amplitudes are added - as vectors, their square norms are measured - as real numbers). At the same time and for the same reason, quantum objects so defined

$\overline{11}$ Situations with regard to which Bohr was lead very early on to introduce the concept of complementarity (in the sense of a complementarity specific to the quantum object, which could manifest itself, according to the type of measurement performed, either as particle or as a wave), which was the object of many controversies.

12 These differences between the notions of incompleteness, as meant by EPR, and of indetermination make our conceptual analysis concerning incompleteness, from Goedel to EPR, very different from the technical correlation, à la Chaitin, beween Goedel's theorem and quantum indetermination in [CaludeStay05].

${ }^{13}$ For example, with inseparability, everything seems to occur as if an event locally well defined in the state space of the definition of quantum magnitudes - an Hilbert space typically, was to potentially project itself upon two distinct points in our usual state space. 
(wave function or state vector, for example) are no longer endowed with the "natural" order structure associated to real numbers (a total order).

ii) Then, as we have seen, the definition of the observables makes it so that some among them (corresponding to the conjugated magnitudes) are not commutable, as opposed to the classical case. This, in the context of the geometrization of this physics, necessitates the introduction of a geometry that is itself non-commutative, thus breaking, as we said, with all previous traditions [Connes94].

iii) Finally, the enquiry may lead even further, with regard to the relationships between quantum phenomenality and the nature of our usual geometric space. May the latter be Newtonian or Riemannian, it will admit a representation as a set of points and its continuum stems from an indefinite divisibility. Now given the existence of a scale of length (possibly minimal, cf. [Nottale99]) such as the Plank length, recent string theories lead to ask if in fact this space would not escape a description in terms of punctual elementarity (eventually to the benefit of another, in terms of interval elementarity $^{14}$, or in terms of higher dimensionality such as "branes").

It should be clear that these specific issues lead to a conceptual revolution in our relating to physical space, at least the space of microphysics. The key idea is that geometry, as a human construction, as we stressed in part, is the consequence of the way we access to space, possibly by measure. So Euclid started by accessing, measuring, with rule and compass. Riemann analyzed, more generally, the rigid body (and characterized the spaces where this tool for measure is preserved: those of constant curbature). Finally, todays noncommutative geometry, in Connes approach, begins by reconstructing space by quantum measure. In microphysics, this happens to be noncommutative (measure this and, later, that, is not equivalent to measuring that and, later, this). And this takes us very far from the space of senses or even classical/relativistic spaces. In conclusion, measure, by rule and compass, by the rigid body, or quantum measure is the form of access we have to space and events in it. This access may differ, yet it may provide a geometric way to a novel unity, by explaining first how to pass from one mathematical organization/understanding of space to another.

\section{Constitutions of objectivity. Conceptual comparisons between mathematics and physics.}

We will now try to understand some aspects of the contingent constitution of mathematical idealities, in analogy to some physical processes, the phase transitions.

\footnotetext{
$\overline{{ }^{14} \text { We would }}$ then maybe pass from a Cantorian representation of continuity to a representation by interval interlockings such as proposed by Veronese or to the nil-potent infinitesimals, see [Bell98].
} 
"Actual infinity", once conceptually constructed by constitutive intersubjectivity, is not only conducive to imagining the idea of God (and theology) or, like Giordano Bruno, the infinity of worlds. It is, on one side, a mathematical concept, and on the other it is involved in the process of constitution of the mathematical objects themselves (both finitary and infinitary). The idea is that mathematical objects are "substantially" (if we may say so) a concentrate of actual infinity brought into play by human beings, within their symbolic culture. Let's explain and argue.

The transcendental constitution of mathematical objectivities (from the finitary ones which are the triangle or the circle, to the structures of well order, or even to the categories of finite objects of which we have spoken) actually involves a very fundamental change of level: the process of abstraction of acts of experience and of the associated constructions (see part I) leads to this transition, which constitutes the forms thus produced, into abstract structures as eidetic objects. This transition (which also leads to their conditions of possibility) presents all the characteristics we ascribe to "physical criticality" (the theory of "critical phase transitions", [Binney92], [Jens98]). Notably, this describes the passage from the local (such or such empirical form) to the global (the structure which is defined abstractly and which is to be found in all particular manifestations), as well as the passage from a certain ("subjectivizing") heteronomy to an autonomy (objectivity), and from a certain instability (circumstantial) to a stability (a-evential). For example, even Euclid's passage from the empirical practice of lines, in measuring "geo", to the concept of thickless line or o dimensionless point, is a conceptual transition, which may be better specified, in our view, in analogy to the critical ones in physics. It is namely these constituted characteristics, resulting from a sort of passage to the effective infinite limit as process of constitution, which lead to mathematical platonic thinking. The latter though, as much as the formal axiomatic approach, forgets the constitutive process itself.

The examples we can use to try to account for this conceptual transition, which leads to the constitution of new objectivities, are varied. In mathematics, we recall the example of the sum of rational numbers $(1 / n !$, for example) which gives, at the actual infinite limit, a transcendental number $(e$, in this case). This may be seen as a critical transition, or "space transition", which leads from one field (the rationals, $\mathbb{Q}$ ) into another one encompassing it (the reals, $\mathbb{R}$ ). In physics, we may find an equivalent in a change of phase, associated to the divergence of an intensive magnitude of the system (a susceptibility, for example, which formally is considered to go to infinity) and to the passage from local to global (divergence of the correlation length of interactions). In biology, it would be a case of a change in the level of functional integration and regulation (the organism in relation to its constituents, for example).

If mathematical structures are also the result of the search for the most stable invariants, as is conceptually characterized in the preceding, it is then probably due, at least in part, to this process of constitution mobilizing a form of actual infinity and leading to a sort of stable autonomy, at the limit. Let's 
continue with the physical metaphor of phase changes. A phase transition can be manifested for instance in a symmetry breaking of the system and a concomitant change (sudden or more progressive) of an order parameter (the total magnetic moment for a para-ferromagnetic transition, density for a liquid/solid transition). In fact, the phase transition is, in a way or another, a transition between disorder (relative) and order (also relative). If we keep these characteristics in mind and make them into a conceptual trait that is common to the transcendental constitution of mathematical objectivities, we will readily notice the disordered situation with regards to the often uncoordinated collection of "empirical" mathematical beings, and the ordered situation in mathematical objects and structures as such, as resulting from the process of abstraction and of constitution.

So it is easily conceivable why the axiomatization, or even the logicization of the statements characterizing these mathematical structures, are genetically and in some respects conceptually second relatively to the mathematical activity and to the process of constitution itself, as we emphasize in the first part of this paper. In our view, here are the roots of the mathematical incompleteness of formalisms. Indeed, the formal statements describe order consecutively to the "phase transition" we have just invoked. But mathematical thinking concerns as much the process of transition as the putting into form and description of its result. And to go even further, we could almost say that the evacuation of the "contingent disorder" accompanying transitionconstitution corresponds to the evacuation of the "significations" associated to the structures over the course of their elaboration and to the "infinitary involvement" it presupposes. This is why the purely logico-formal foundations lead to pure syntaxes devoid of meaning and intrinsically incomplete.

It is probably also one of the reasons enabling to understand why formalism "works" when it is an issue of describing the order resulting from the transition in question (the constituted mathematical structures), but that it fails from the moment it is given the task of also describing the transition itself, that is, the process of constitution as such, from and in the terms of its result. In fact, one may consider that formalism fails to capture "actual infinity" that enabled the passage and which has become a major characteristic of the objectivities thus constructed. In this sense, the "semantic" aspect is the most deeply involved in the occurrence of this effective passage to the infinite limit, whereas the syntactic aspect is much more relative to the rigorous, possibly axiomatic, description of the once stabilized results of this passage. We insist that it is the non-coincidence of these two dimensions that is at the origin of the properties of incompleteness, that we saw as a discrepancy or gap between construction principles (conceptual) and proof principles (formal), of which we speak in part I. The results of incompleteness are a demonstration of these lackings.

So here ends, in our view, the proposed conceptual analogy with phase transitions in physics because we know that in physics, as we have recalled earlier, renormalization theory proves itself in a way to be able to address the 
critical transition itself. This difference in behavior relatively to the processes put into play is to be referred to the difference between the objects considered themselves, such as they are elaborated in physics and in mathematics: if the construction principles are similar, as we have shown in [BaillyLongo06] and hinted here, the proof principles are completely different (empirical vs. formal), and it is indeed regarding the status of the proof that the difference is manifest.

It is probably what transpires in this dichotomy introduced in [BaillyLongo06] relatively to elementarity. As a matter of fact, we opposed the elementary and simple (related to the artificial processes of algorithmic calculus, to the concatenation of simple logical gates, or even to any artifact) to the elementary and complex (related to natural processes such as strings in quantum physics or cells in biology). Quantum physics and biology address natural phenomena that are confronted to elementarities that are rather complex and hence they seem irreducible to processes grounded on simple elementarities, in the sense of artificial computation and general artefacts. Besides the role of meaning in deduction (a role stressed by "concrete" incompleteness in particular, see [Longo02]), this further prevents from reducing scientific judgment to a calculus, in this sense, without denying, of course, the interest of the complementary understanding provided by the formal and computational descriptions.

\section{Conclusion}

Let's conclude this paper by stressing the perspective that guided our work. In our views, the epistemological investigation of mathematics cannot be detached from a constitutive analysis of concepts and structures (and thus of the very object of knowledge) in other scientific disciplines, such as physics. This is the project that, in a very preliminary and modest fashion, but along the same phenomenological approach, we try to pursue in several papers and in [BaillyLongo06], an extension, within a scientific project, of some of the ideas we hinted here. The analogies and differences in the "phenomenology of incompleteness" is a fundamental part of it. We believe that further work should lead to an analysis of this phenomenon in other disciplines (see [LongoTe07] for some reflections on a form of causal incompleteness in biology).

\section{Acknowledgements}

We would like to thank the anonimous referees for their many comments and questions. Longo's papers are downloadable: http://www.di.ens.fr/users/longo/ 


\section{References}

[Aspect82] Aspect A., Grangier P. and Roger G., Experimental Realization of the Einstein-Podolsky-Rosen-Bohm Gedankenexperiment : A New Violation of Bell's Inequalities Phys. Rev. Let. 49, p.91, 1982.

[BaillyLongo06] Bailly F., Longo G., Mathématiques et sciences de la nature. La singularité physique du vivant. Hermann, Paris, 2006 (English introduction, downloadable).

[BaillyLongo07] Bailly F., Longo G. Randomness and Determination in the interplay between the Continuum and the Discrete, Mathematical Structures in Computer Science, vol. 17, n. 2, 2007.

[Barreau89] Barreau H. et Harthong J. (Eds.), La mathmatique non standard, Ed. CNRS 1989.

[Barrow97] Barrow-Green J., Poincare' and the three body Problem, AMS, 1997.

[Bell98] Bell J., A Primer of Infinitesimal Analysis, Cambridge U.P., 1998.

[Bell64] Bell J.S., On the Einstein-Podolsky-Rosen Paradox Physics 1, p.195, 1964.

[Binney92] Binney J., Dowrick N.J., Fisher A.J., and Newman M.E.J., The Theory of Critical Phenomena: An Introduction to the Renormalization Group. Oxford University Press, Oxford, 1992.

[Bitbol00] Bitbol M. L'aveuglante proximité du réel, Flammarion 2000.

[Bohm51] Bohm D., The Paradox of Einstein, Rosen and Podolsky Quantum Th. p.611, 1951.

[BrattkaPresser03] Brattka V., Presser G., Computability on subsets of metric spaces, Theoretical Computer Science, 305, 2003.

[BrattkaWeihrauch99] Brattka V., Weihrauch K., Computability on subsets of Euclidean space I: Closed and compact subsets, Theoretical Computer Science, 219, 1999.

[Calude02] Calude C. Information and Randomness: An Algorithmic Perspective, Springer, Berlin, 2002.

[CaludeStay05] Calude C., Stay M., From Heisenberg to Goedel via Chaitin. International Journal of Theoretical Physics, vol. 44, 7, pp. 1053-1065, 2005.

[Collins05] Collins P., Continuity and computability of reachable sets. Theoretical Computer Science, 341, 2005.

[Connes94] Connes A., A. Non-commutative Geometry, Academic Press, 1994.

[Dehaene97] Dehaene S. The Number Sens, Oxford U.P., 1997.

[Devaney89] Devaney R. L. An introduction to Chaotic Dynamical Systems, Addison-Wesley, 1989.

[Diacu92] Diacu F. Singularities of the N-Body Problem, Publications CRM, Montreal, 1992.

[Einstein35] Einstein A., Podolsky B. and Rosen N., Can Quantum-Mechanical Description of Physical Reality be Considered complete ? Phys. Rev. 41, P.777, 1935.

[vanFrassen] van Frassen B., Lois et symetries, Vrin, Paris, 1994.

[Frege84] Frege G., The Foundations of Arithmetic, 1884 (Engl. transl. Evanston, 1980.)

[Goedel49] Gödel K., Remark About the Relationship between Relativity Theory and Idealistic Philosophy in P.A.Schilpp (ed.), Albert Einstein: Philosopher-Scientist, LaSalle IL, Open Court, pp. 557-562, reprinted with corrections and additions in Collected Works, S. Feferman et. al. (eds.), Vol. 2, Oxford University Press, Oxford. 
[Harrington85] Harrington L. et al. (eds) H. Friedman's Research on the Foundations of Mathematics, North-Holland, 1985.

[Hilbert99] Hilbert D., Les fondements de la géométrie, 1899 (trad. fran., Dunod, 1971).

[Hoyrup07] Hoyrup M., Dynamical Systems: Stability and Simulability, Mathematical Structures in Computer Science, vol. 17, n. 2, 2007.

[Hoyrup08] Hoyrup M., Kolcak A., Longo G. Computability and the Morphological Complexity of some dynamics on Continuous Domains Invited survey TCS, to appear, 2008.

[Jech97] Jech T., Set Theory, Springer, 1997.

[Jens98] Jensen H. J., Self-Organized Criticality, Emergent Complex Behavior in Physical and Biological Systems, Cambridge Lectures in Physics, 1998.

[Laskar94] Laskar J., Large scale chaos in the Solar System. Astron. Astrophysics, 287, L9 L12, 1994.

[Longo01] Longo G. Laplace (A note on incompleteness). An item of J-Y. Girard's Logic Dictionnary, at the end of "Locus Solum", MSCS, vol.11, n.3, Cambridge Univ. Press, 2001.

[Longo02] Longo G. "Reflections on Incompleteness or On the proofs of some formally unprovable propositions and Prototype Proofs in Type Theory" Invited Lecture, Types for Proofs and Programs, Durham, (GB), Dec. 2000; Lecture Notes in Computer Science, vol 2277 (Callaghan et al. eds), pp. 160 - 180, Springer, 2002.

[Longo05] Longo G., The Cognitive Foundations of Mathematics: human gestures in proofs and mathematical incompleteness of formalisms, in Images and Reasoning, (M. Okada et al. eds.), Keio University Press, Tokio, 2005.

[Longo07] Longo G., Laplace, Turing and the "imitation game" impossible geometry: randomness, determinism and programs in Turing's test. In Epstein, R., Roberts, G., \& Beber, G. (Eds.). The Turing Test Sourcebook. Dordrecht, The Netherlands: Kluwer, 2007.

[LongoTe07] Longo G., Tendero P.E., The causal incompleteness of Programming Theory in Molecular biology, to appear in Foundations of Science. A french version is in Evolution des concepts fondateurs de la biologie du XXIe siècle, (Miquel ed.) DeBoeck, Paris, 2007.

[LongoPaul08] LongoG., Paul T., The Mathematics of Computing between Logic and Physics, to appear in "Computability in Context: Computation and Logic in the Real World", (Cooper, Sorbi eds.), Imperial College Press/World Scientific, 2008.

[ML66] Martin-Löf P. The definition of random sequences, Information and Control 9: 602619, 1966.

[Nottale99] Nottale L., La relativité dans tous ses états, Hachette, 1999.

[ParisHarr78] Paris J., Harrington L., A mathematical incompleteness in Peano Arithmetic, Handbook of Mathematical Logic, Barwised ed., 1978.

[Patras01] Patras F., La pense mathmatique contemporaine, PUF 2001.

[Petersen90] Petersen K. Ergodic Theory, Cambridge University Press, 1990.

[Petit96] Petit J.-L., Solipsisme et Intersubjectivité, Cerf,1996

[Pilyugin99] Pilyugin S. Yu., Shadowing in dynamical systems, Springer, Berlin, 1999.

[PourElRi89] Pour-El M.B., Richards J.I., Computability in analysis and physics. Perspectives in mathematical logic, Springer, Berlin, 1989. 
[Salanskis91] Salanskis J-M., L'hermneutique formelle, Ed. CNRS, 1991.

[Tappenden95] Tappenden J., Geometry and generality in Frege's philosophy of Arithmetic. Synthese, n. 3, vol. 102, March 1995.

[Tieszen05] Tieszen R.., Phenomenology, Logic, and the Philosophy of Mathematics, Cambridge U. P., 2005

[Weyl49] Weyl H., Philosophy of Mathematics and of Natural Sciences, 1927 (english transl., Princeton University Press, 1949).

[Wey00] Weihrauch K., Computable Analysis. Texts in Theoretical Computer Science. Springer, Berlin, 2000. 